\title{
Near-field imaging of spin-locked edge states in all-dielectric topological metasurfaces
}

Cite as: Appl. Phys. Lett. 114, 031103 (2019); https://doi.org/10.1063/1.5055601

Submitted: 08 September 2018 . Accepted: 21 December 2018 . Published Online: 23 January 2019

Alexey Slobozhanyuk, Alena V. Shchelokova (D), Xiang Ni, S. Hossein Mousavi, Daria A. Smirnova, Pavel A. Belov, Andrea Alù, Yuri S. Kivshar, and Alexander B. Khanikaev

ARTICLES YOU MAY BE INTERESTED IN

Experimental observation of a photonic hook

Applied Physics Letters 114, 031105 (2019); https://doi.org/10.1063/1.5065899

Room temperature surface emission on large-area photonic crystal quantum cascade lasers Applied Physics Letters 114, 031102 (2019); https://doi.org/10.1063/1.5082279

Magnetic-field tailoring of the terahertz polarization emitted from a spintronic source Applied Physics Letters 114, 031101 (2019); https://doi.org/10.1063/1.5055736

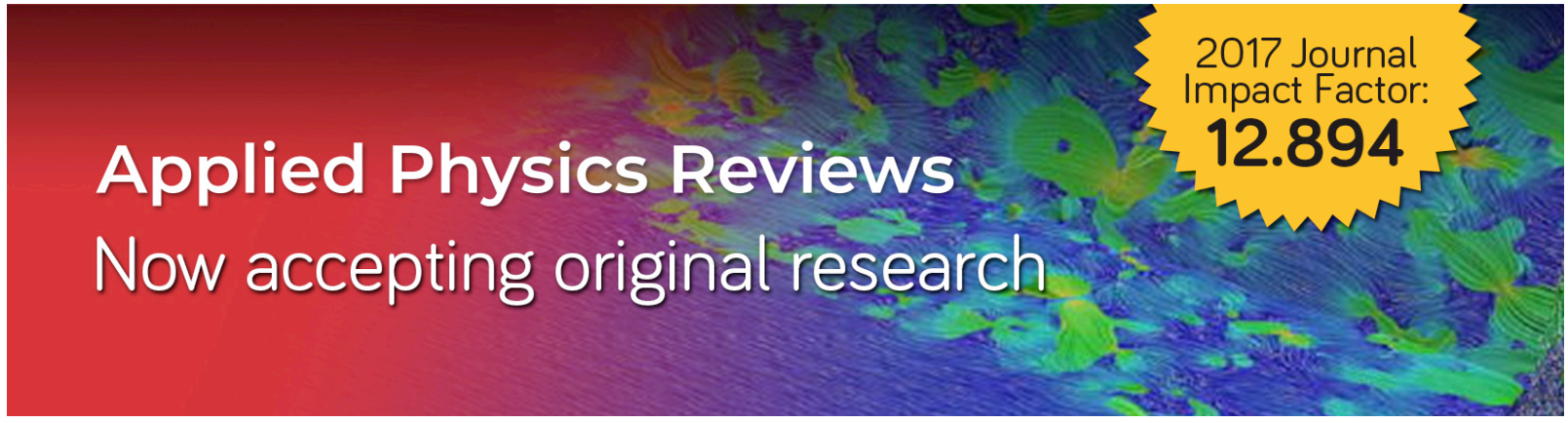




\title{
Near-field imaging of spin-locked edge states in all-dielectric topological metasurfaces
}

\author{
Cite as: Appl. Phys. Lett. 114, 031103 (2019); doi: 10.1063/1.5055601 \\ Submitted: 08 September 2018 - Accepted: 21 December 2018 - Published Online: \\ 23 January 2019
}

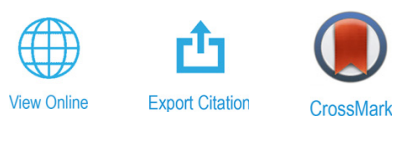

Alexey Slobozhanyuk, ${ }^{1,2, a)}$ Alena V. Shchelokova, ${ }^{1}$ (D) Xiang Ni, ${ }^{3,4}$ S. Hossein Mousavi, ${ }^{5}$ Daria A. Smirnova, ${ }^{2,3}$ Pavel A. Belov, ${ }^{\top}$ Andrea Alù, ${ }^{5}$ Yuri S. Kivshar, ${ }^{1,2}$ and Alexander B. Khanikaev ${ }^{1,3,4, a)}$

\author{
AFFILIATIONS \\ ${ }^{1}$ Department of Nanophotonics and Metamaterials, ITMO University, St. Petersburg 197101, Russia \\ ${ }^{2}$ Nonlinear Physics Centre, Australian National University, Canberra, ACT 2601, Australia \\ ${ }^{3}$ Department of Electrical Engineering, Grove School of Engineering, The City College of the City University of New York, \\ 140th Street and Convent Avenue, New York, New York 10031, USA \\ ${ }^{4}$ Graduate Center of the City University of New York, New York, New York 10016, USA \\ ${ }^{5}$ Microelectronics Research Centre, Cockrell School of Engineering, University of Texas at Austin, Austin, Texas 78758, USA \\ a) Electronic addresses: a.slobozhanyuk@metalab.ifmo.ru and khanikaev@gmail.com
}

\begin{abstract}
A new class of phenomena stemming from topological states of quantum matter has recently found a variety of analogies in classical systems. Spin-locking and one-way propagation have been shown to drastically alter scattering of electromagnetic waves, thus offering an unprecedented robustness to defects and disorder. Despite these successes, bringing these new ideas to practical grounds meets a number of serious limitations. In photonics, when it is crucial to implement topological photonic devices on a chip, two major challenges are associated with electromagnetic dissipation into heat and out-of-plane radiation into free space. Both these mechanisms may destroy the topological state and seriously affect the device performance. Here, we demonstrate experimentally that the topological order for light can be implemented in all-dielectric on-chip prototype metasurfaces, which mitigate the effect of Ohmic losses by using exclusively structured dielectric materials, and we reveal that coupling of the system to the radiative continuum does not affect topological properties. We demonstrate the spin-Hall effect of light for spinpolarized topological edge states through near-field spectroscopy measurements.
\end{abstract}

Published under license by AIP Publishing. https://doi.org/10.1063/1.5055601

Theoretical predictions ${ }^{1-12}$ and experimental realizations $^{13-22}$ of photonic topological systems have demonstrated fascinating properties for their topology-preserved electromagnetic modes and opened avenues for their use in practical systems and devices. ${ }^{23}$ Unfortunately, many of these original proposals have been based on metal-containing metamaterials, ${ }^{24-28}$ whose functionalities are limited by Ohmic losses. In this respect, dielectric topological structures based on arrays of ring resonators ${ }^{15,29,30}$ or chiral waveguides ${ }^{16,31,32}$ have demonstrated desirable properties. Nonetheless, this comes at the cost of a very large footprint, with arrays of resonators that can be hundreds to thousands of wavelengths in size, rendering such systems rather unpractical. It is of practical relevance to realize topological systems not only made of photonic compatible materials but also compact enough for integration with modern photonics circuitry. In this case, one has to consider structures with characteristic length scales smaller ${ }^{33}$ or comparable in size $^{34}$ with the wavelength of light. In this regard, all-dielectric metamaterials and metasurfaces ${ }^{35-39}$ have recently emerged as a well-suited platform to realize compact topological photonic insulators. Based on this concept, here we implement alldielectric topological metasurface, demonstrate topologically robust photonic transport, and visualize directly the robust topological edge states revealing their spin-locking properties by near-field spectroscopy.

The structure that we study is schematically shown in Fig. 1(a), and it represents a triangular array of dielectric disks with their dimensions tuned such that the array supports two doubly degenerate Dirac cones at $\mathrm{K}$ and $\mathrm{K}^{\prime}$ points of the Brillouin zone. The band structure plotted in Fig. 1(b) reveals two pairs of the Dirac bands, stemming from electric and magnetic dipolar modes of the disks, respectively. The corresponding dipolar 


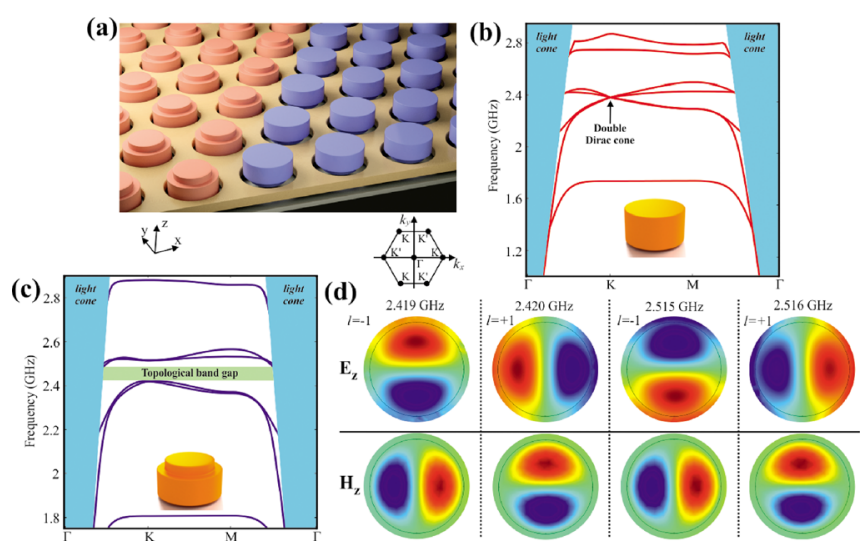

FIG. 1. (a) Geometry of a metasurface with a domain wall between arrays that possess different topological characteristics. The period is $36 \mathrm{~mm}$, the large disk radius and height are $14.57 \mathrm{~mm}$ and $9 \mathrm{~mm}$, and the small disk radius and height are $11 \mathrm{~mm}$ and $3 \mathrm{~mm}$, respectively. Photonic band structure of the metasurface without (b) and with (c) bianisotropy. Insets in panels (b) and (c) show the corresponding meta-atoms of both metasurfaces. The green shaded area illustrates the spectral width of the topological bandgap. The position of the light line is marked by a blue shaded area. (d) Field profiles of eigenmodes of the bianisotropic metasurface, revealing locking of their electric and magnetic field components.

modes, carrying angular momentum $l= \pm 1$, are polarized in the $x$ - and $y$-directions. The value of angular momentum is related to the dipolar nature of the modes, which correspond to the $l=1$ and $l=-1$ multipole expansion, both for the electric and magnetic modes, respectively. The presence of the Dirac points stems from the degeneracy of these modes at $\mathrm{K}$ and $\mathrm{K}^{\prime}$ points due to PT and the $\mathrm{C}_{3}$ rotational symmetries of the lattice.

The double degeneracy between electric and magnetic dipolar modes enables emulating the spin degree of freedom of topological insulators. ${ }^{40,41}$ On the other hand, coupling between electric and magnetic modes allows emulating coupling of spin and orbital degrees of freedom, essentially the spin-orbit interaction of light. ${ }^{9,42}$ To induce such coupling, we use the fact that electric and magnetic modes have different parity in the out-of-plane (z) direction, and we break this symmetry by introducing a circular notch on one of the flat faces of the cylinders. This gives rise to hybridization of the electric and magnetic dipolar modes, opening a complete photonic bandgap [Fig. 1(c)]. Alternatively, to break inversion symmetry, one can also consider a symmetric dielectric cylinder with a slit. ${ }^{43} \mathrm{~A}$ similar mechanism to induce bianisotropic response was employed recently in metallic structures ${ }^{25}$ and phononic ${ }^{44}$ systems. Note that, while this gap is complete below the light line, eigenmodes located above the light line may couple to the continuum of radiation in the cladding due to the finite thickness of the structure. Nonetheless, the top and bottom bands do not cross anywhere in the Brillouin zone, which implies that the topological properties are completely defined by the hybridization of magnetic and electric Dirac bands near the Kand $K^{\prime}$ points.

The eigenmodes of the metasurface with bianisotropy no longer represent pure electric and magnetic modes but are mixed states with phases between their electric and magnetic components being locked, as shown in Fig. 1(d). The dipolar

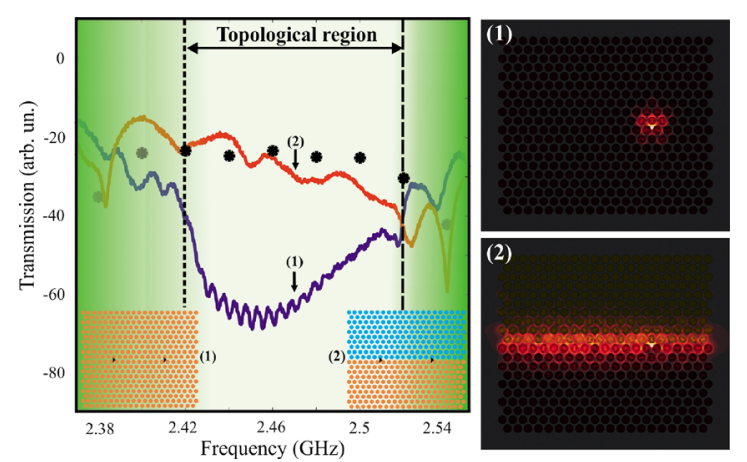

FIG. 2. Experimental transmission spectra of the topological metasurface without the domain wall (blue line), along with the domain wall (red line) and along the domain wall with sharp corners (red dashed line). Location of the dipolar sources (black triangles) and geometrical configurations used in experiments are shown in the insets. Black stars correspond to the numerically calculated values of the transmission in the case of the domain wall. The right panels show the distribution of the electric field amplitude at the frequency of $2.468 \mathrm{GHz}$ for two different configurations of the metasurfaces, without the domain wall (1) and with the domain wall (2) which supports the topological edge states.

electric and magnetic moments of these modes always appear to be tilted by $90^{\circ}$ and $-90^{\circ}$ with respect to each other. These mixed states are referred to as pseudo spin-up $(\uparrow)$ and spin-down $(\downarrow)$ states and appear pairwise as top and bottom bands in the spectrum. To confirm that these new states possess topological properties, we performed numerical calculations of the topological invariant (see supplementary material) and found that the upper and lower modes are characterized by non-vanishing spin-Chern numbers $C_{l / u}^{\uparrow}= \pm 1$ and $C_{l / u}^{\downarrow}=\mp 1$, where the subscripts indicate lower (l) and upper (u) bands. Importantly, the reversal of the orientation of the meta-atoms in the vertical direction leads to the reversal of the pseudo-spins and of the bands in the spectrum, which is also accompanied by an inversion of the topological invariant to $C_{l / u}^{\uparrow}=\mp 1$ and $C_{l / u}^{\downarrow}= \pm 1$, respectively.

The proposed structure was fabricated using high index $(\epsilon=39)$ ceramic discs (see supplementary material). The particles have been arranged into a triangular array and the collective response was studied by RF spectroscopy. First, we confirmed that the transmission spectra of array possess a bandgap induced by the bianisotropy in the frequency range of 2.42-2.52 GHz (Fig. 2). Our measurement results are in full agreement with the numerical calculations of the band structure (see the right panel in Fig. 2).

The major interest in topological electromagnetic phases lies in the fact that they support robust edge states at their interfaces. As an interface, we choose a domain wall separating two topologically distinct domains of the metasurface. The two domains represent crystals with opposite orientation of the meta-atoms in the vertical direction, thus effectively creating a topological interface, across which the spin-Chern numbers experience a change of magnitude 2 . The bulk-interface correspondence ${ }^{9}$ implies that such a domain wall should host a total of four states, two with spin-up and two with spin-down, respectively.

The numerical simulations carried out for a supercell of 24 meta-atoms with a domain wall in the middle confirm the 

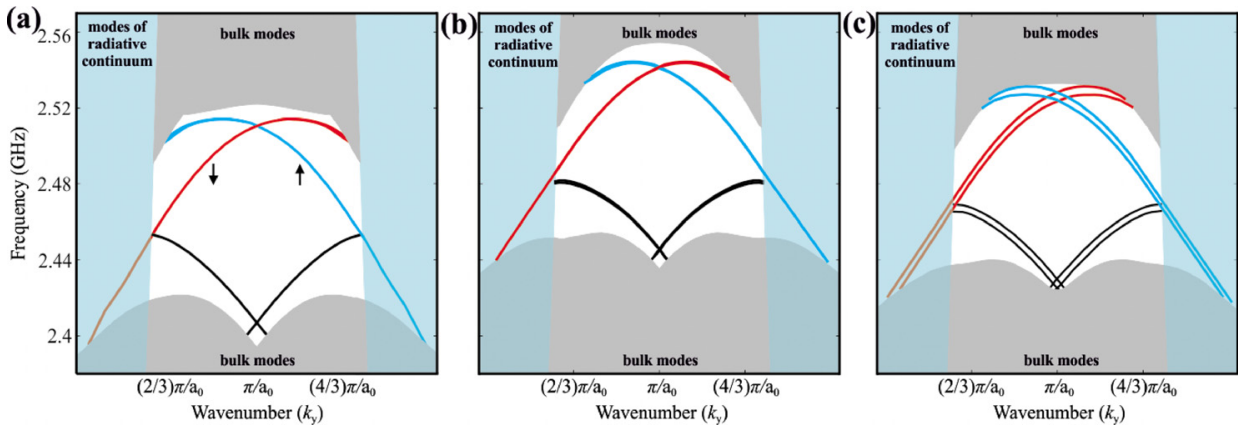

FIG. 3. Dispersions of the photonic topological edge states supported by the domain wall in the case of the metasurface with optimal geometrical parameters (a) and suboptimal parameters (b) and the metasurface where the disorder in duality is introduced (c). The grey shaded areas illustrate the bulk photonic states, while the blue shaded area corresponds to the modes of radiative continuum. The black arrows show the spin-up and spin-down modes. The metasurface with suboptimal parameters has the following parameters: the period is $36 \mathrm{~mm}$, the large disk radius and height are $14.23 \mathrm{~mm}$ and $9 \mathrm{~mm}$, and the small disk radius and height are $10.7 \mathrm{~mm}$ and $3 \mathrm{~mm}$, respectively. The disorder in duality was achieved through random distribution of the diameter of the dielectric cylinders with magnitudes of dispersion of $2 \%$.

existence of edge states. The edge states appear within the topological bandgap and are strongly localized to the domain wall in the lateral direction, and while they do lay below the light cone, they apparently overlap spectrally with the modes of radiative continuum laying above the light line (Fig. 3). Despite this, the edge modes have negligible leakage to the continuum. Importantly, no such localized edge modes have been found for similar calculations carried out for the case of all meta-atoms facing up, while an identical bulk spectrum was obtained (not shown), thus confirming the topological origin of the edge states.

Interestingly, only two of the modes appear to cross the entire topological bandgap, while the second pair touches the light line and disappears in the middle of the gap after emerging from the continuum of the bottom bulk modes. This is important distinction of the open metasurface from its closed photonic counterparts in which 4 topological edge modes would also cross the entire bandgap due to the absence of the light cone and the continuum of modes within it. ${ }^{9}$ The inspection of the field profiles for the two sets of modes shows that those crossing the gap represent a set of even modes, symmetric across the domain wall (see supplementary material). These modes can be classified as spin-up and spin-down (as shown by the arrows) by inspecting the power flux inside the particles in the interface proximity, e.g., the spatial profile of the forward-propagating edge mode is clockwise-rotating in the left domain and counter-clockwise-rotating in the right domain. This feature is consistent with spin-polarized photon transport in the forward direction (see supplementary material and Ref. 9). Note that for the backward-propagating spin-down mode, the sense of rotation is reversed. For these modes, one spin-up and one spindown, the coupling to radiative continuum is largely suppressed by their symmetry, which ensures that such coupling does not destroy the topological nature. Such suppressed coupling can be understood by noticing that these modes have a quadrupolar $\mathrm{Q}_{x 2-y 2}$ profile which has very low radiation efficiency. Meanwhile, the odd edge states not only cross the light cone but appear to interact strongly with the radiative continuum affecting their dispersion by pushing bands to the low frequency range-a clear indication of destruction of their topological properties caused by the interaction with the continuum. This strong interaction of the odd edge modes with the continuum can also be understood from their field profile across the domain wall (see supplementary material), which has a clear out-of-plane magnetic dipolar $\mathrm{M}_{\mathrm{z}}$ (and quadrupolar $\mathrm{Q}_{x y}$ ) configuration and efficiently radiates in the direction close to lateral. More importantly, however, this mode also has negative group velocity. The band crossing edge state, in contrast, has a very small dipole moment and thus radiates less efficiently, but, due to its positive group velocity, it remains confined to the topological bandgap at higher frequencies, which allows it to cover the entire bandgap region. Another important difference we should consider is related to the fact that the dipoles are not isolated, but periodic along the domain wall. Therefore, it is important to consider the momentum of the modes along the domain wall. Thus, the $\mathrm{Q}_{x 2-y 2}$ mode radiates along the domain wall with proper phase velocity, which facilitates guiding of the edge state. The profile of the $Q_{x y}$ mode, in contrast, tends to radiate in the direction perpendicular to the domain wall, which leads to stronger leakage of the mode.

It is instructive to check the robustness of topological edge states in the case of violation of the duality symmetry. The suboptimal design of cylinders leads to the removal of the degeneracy between electric and magnetic dipolar modes, and duality symmetry is broken (see supplementary material). Figure 3(b) shows that the edge modes maintain their gapless character, as long as the spectral detuning between the Dirac cones remains smaller than the topological gap open by the bianisotropy. In addition to the robustness against uniform violation of the duality, a randomized distribution of such symmetry reduction was introduced over the entire supercell. As seen from Fig. 3(c), while the bulk bandgap expectedly narrows, the topological edge modes maintain their gapless character. In fact, the only modification to the band diagram is the slightly different dispersion of the edge modes localized to the two opposite domain walls of the supercell, which perceive different effective environments.

To experimentally confirm the existence, spin-locked propagation, and robustness of the topological edge states, we 

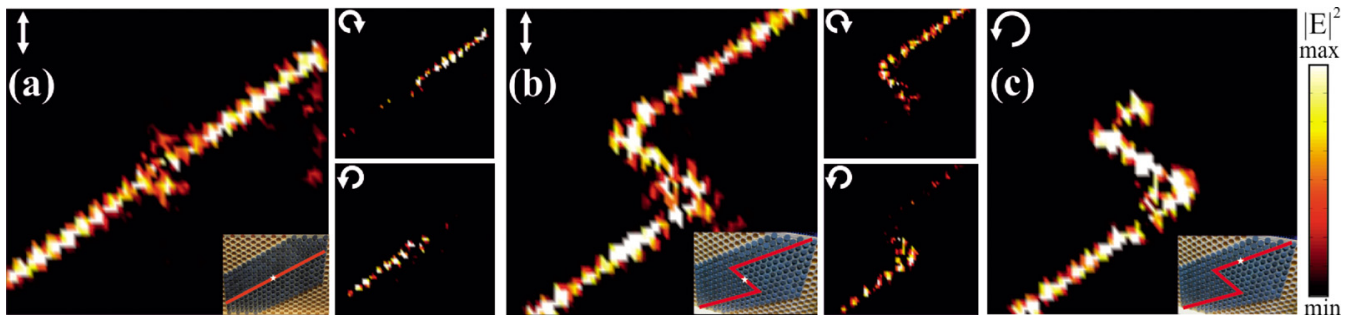

FIG. 4. Experimentally measured electric near-field intensity images for the linear (a) and double-bend (b) and (c) domain walls at the frequency of $2.462 \mathrm{GHz}$. The insets show photographs of the metasurfaces with the location of the domain wall indicated by the red line and the source position marked by the star symbol. The white arrows denote the polarization of the source.

changed the configuration of the array such that half of the meta-atoms were oriented upward and the second half downward, thus forming the domain wall across the straight line. The edge modes were then excited by a dipole antenna placed in the middle of the domain wall in the horizontal direction and just above the array to ensure coupling to the near field. The presence of the topological edge mode was tested by placing the dipole source at the domain wall and the transmission spectrum was measured (Fig. 2). As expected, enhanced transmission within the bandgap region occurred due to the excitation of the edge mode. A similar behavior was observed in numerical simulations (see black stars in Fig. 2). The precise location of the antenna allowed us exciting the topological edge states with the electric field maximum at the center of the domain wall. At the same time, controlling the polarization of the dipole antenna enables selective excitation of the mode with specific angular momentum. The edge states appear to have opposite angular momentum in the two adjacent domains. Thus, while the pseudo-spin of the edge-state is unique, in two domains, it is locked to opposite angular-momenta. However, at the same time, there is an asymmetry in field distribution in the vertical direction due to the introduced notches. For this reason, by approaching the domain wall on one side of the crystal and at the same time slightly off the mid-point of the domain wall, it is possible to couple the dipolar antenna source to a predominantly circular $(l=1$ or $l=-1)$ rotating pattern of the edge mode. This property facilitates the directional excitation of edge states based on the relation between pseudo-spin and angular momentum in a particular domain and the pseudo-spin and the propagation direction in the entire structure. Thus, circular left or right polarization excites one of the two particular pseudospins due to the locking of spin and angular momentum degrees of freedom. As it can be seen from the map of the near field collected by another antenna attached to the xy-motorized stage, the linear polarization excites edge modes of both spins running in two opposite directions away from the source [Fig. 4(a)]. However, switching to circular polarization changes the field pattern and left- and right-handed excitations result in a unidirectional flow of radiation from the source [Fig. 4(a)]. These experimental results unambiguously confirm the spin-locking character of the edge states.

The observed spin-locking is known to be responsible for the unprecedented robustness of the edge states to structural imperfections and defects. Here, we expect to see this robustness in an all-dielectric platform, while the open geometry of the system offers additional benefits by allowing us to directly visualize reflectionless flow of the field by the near-field measurements. To confirm such robustness, we created a domain wall configuration with two sharp bends and carried out experimental measurements. We performed near-field measurements with a source placed in the middle of the central segment. The circularly polarized feed again leads to selective excitation of spin-up or spin-down modes, which flow towards a specific bend. As expected from the topological robustness, the modes flow around the bends without back-reflection [Fig. 4(b)). Similar unidirectional excitation and robust propagation were also found in the case of the source placed in the first long arm of the domain wall [Fig. 4(c)], in which case the spin-up edge state propagates across two sharp bends without back reflection and avoids the formation of standing-wave field-configurations in the central segment.

To summarize, a topological metasurface has been experimentally implemented using all-dielectric materials and tested in the microwave frequency range. Despite the open nature of the system, we found that the system maintains its bulk topological characteristics. The topological interfaces in the form of domain walls were tested and found to host two types of edge states, with only one of them interacting and coupling with the continuum of modes above the light line. As a result, this type of edge state loses its topological characteristics merging with the radiative continuum without crossing the topological bandgap. The second class of edge states is unaffected by the presence of the radiative continuum and exhibits topological properties such as spin-locking and lacks back scattering from defects. The open nature of the system allowed direct visualization of these properties, unambiguously confirming the topological robustness of electromagnetic transport along the domain wall.

In the optical domain, the proposed all-dielectric metasurface can be implemented with the use of silicon or germanium (see supplementary material). As was shown experimentally, the geometry of silicon nanodisks may be optimized to achieve degenerate magnetic and electric dipolar responses in order to produce Huygens' metasurfaces, ${ }^{35}$ whereas an asymmetry in the meta-atom design can be introduced in order to reach bianisotropic response. ${ }^{43}$ This effect can be also achieved or further modified/tuned by substrate-induced bianisotropy. ${ }^{45,46}$ In our microwave experiment, the substrate also breaks the z-mirror symmetry, and this results in a narrow bandgap opening (see 
supplementary material). However, the effect of the substrate is much weaker than the one produced by the judicious asymmetry in the meta-atom design. If considered detrimental, the effect of the substrate can be reduced by fabricating the metasurface on ultrathin membranes. ${ }^{47}$

The use of dielectric materials and small footprints of the proposed metasurface design envision integration of photonic and electronic components on the same substrate. At the same time, the topological nature of the design provides robust transport and versatile control of optical signals by synthetic gauge fields.

See supplementary material for details of (1) experiments, (2) numerical modelling, (3) optical metasurface design, (4) field profiles of the topological modes, (5) robustness of topological modes, (6) influence of the substrate, (7) measurements in a broad frequency range, and (8) measurements of a single metaatom response.

The authors are grateful to E. Nenasheva for the fabrication of ceramic resonators, and thank D. Filonov for an assistance with experimental measurements at the initial stage of this project. The work was supported by the National Science Foundation (Grant Nos. CMMI-1537294 and EFRI1641069). The experimental part of the work was supported by the Russian Science Foundation (Grant No. 16-19-10538) and numerical calculations were partially supported by the Russian Foundation for Basic Research (Grant No. 18-3220065). The work of AS, DAS, and YSK was partially supported by the Australian Research Council. Research was partly carried out at the Center for Functional Nanomaterials, Brookhaven National Laboratory, which is supported by the U.S. Department of Energy, Office of Basic Energy Sciences, under Contract No. DE-SC0012704.

\section{REFERENCES}

${ }^{1}$ F. Haldane and S. Raghu, Phys. Rev. Lett. 100, 013904 (2008).

${ }^{2}$ L. Lu, J. D. Joannopoulos, and M. Soljaćić, Nat. Photonics 8, 821 (2014).

${ }^{3}$ V. Peano, C. Brendel, M. Schmidt, and F. Marquardt, Phys. Rev. X 5, 031011 (2015).

${ }^{4}$ L. Lu, C. Fang, L. Fu, S. G. Johnson, J. D. Joannopoulos, and M. Soljačić, Nat. Phys. 12, 337 (2016).

5J.-W. Dong, X.-D. Chen, H. Zhu, Y. Wang, and X. Zhang, Nat. Mater. 16, 298 (2017).

${ }^{6}$ K. Y. Bliokh, D. Smirnova, and F. Nori, Science 348, 1448 (2015).

${ }^{7}$ K. Fang, Z. Yu, and S. Fan, Nat. Photonics 6, 782 (2012).

${ }^{8}$ L. Lu, L. Fu, J. D. Joannopoulos, and M. Soljačić, Nat. Photonics 7, 294 (2013).

${ }^{9}$ A. B. Khanikaev, S. H. Mousavi, W. K. Tse, M. Kargarian, A. H. MacDonald, and G. Shvets, Nat. Mater. 12, 233 (2013).

${ }^{10}$ L. - H. Wu and X. Hu, Phys. Rev. Lett. 114, 223901 (2015).

"M. G. Silveirinha, Phys. Rev. B 94, 205105 (2016)

${ }^{12}$ S. Barik, H. Miyake, W. DeGottardi, E. Waks, and M. Hafezi, New J. Phys. 18, 113013 (2016).

${ }^{13}$ Z. Wang, Y. Chong, J. D. Joannopoulos, and M. Soljačić, Nature 461, 772 (2009).
${ }^{14}$ Y. Poo, R. Wu, Z. Lin, Y. Yang, and C. Chan, Phys. Rev. Lett. 106, 093903 (2011).

${ }^{15}$ M. Hafezi, S. Mittal, J. Fan, A. Migdall, and J. M. Taylor, Nat. Photonics 7, 1001 (2013).

${ }^{16}$ M. C. Rechtsman, J. M. Zeuner, Y. Plotnik, Y. Lumer, D. Podolsky, F. Dreisow, S. Nolte, M. Segev, and A. Szameit, Nature 496, 196 (2013).

${ }^{17}$ L. Lu, Z. Wang, D. Ye, L. Ran, L. Fu, J. D. Joannopoulos, and M. Soljačić, Science 349, 622 (2015).

${ }^{18}$ S. A. Skirlo, L. Lu, Y. Igarashi, Q. Yan, J. Joannopoulos, and M. Soljačić, Phys. Rev. Lett. 115, 253901 (2015).

${ }^{19}$ A. P. Slobozhanyuk, A. N. Poddubny, A. E. Miroshnichenko, P. A. Belov, and Y. S. Kivshar, Phys. Rev. Lett. 114, 123901 (2015).

${ }^{20}$ M. I. Shalaev, W. Walasik, A. Tsukernik, Y. Xu, and N. M. Litchinitser, Nat. Nanotechnol. 14, 31-34 (2019).

${ }^{21}$ Y. Yang, Y. F. Xu, T. Xu, H.-X. Wang, J.-H. Jiang, X. Hu, and Z. H. Hang, Phys. Rev. Lett. 120, 217401 (2018).

${ }^{22}$ S. Barik, A. Karasahin, C. Flower, T. Cai, H. Miyake, W. DeGottardi, M. Hafezi, and E. Waks, Science 359, 666-668 (2018).

${ }^{23}$ L. Lu, J. D. Joannopoulos, and M. Soljačić, Nat. Phys. 12, 626 (2016).

${ }^{24}$ W.-J. Chen, S.-J. Jiang, X.-D. Chen, B. Zhu, L. Zhou, J.-W. Dong, and C. T. Chan, Nat. Commun. 5, 5782 (2014).

${ }^{25}$ A. P. Slobozhanyuk, A. B. Khanikaev, D. S. Filonov, D. A. Smirnova, A. E. Miroshnichenko, and Y. S. Kivshar, Sci. Rep. 6, 22270 (2016).

${ }^{26} \mathrm{X}$. Cheng, C. Jouvaud, X. Ni, S. H. Mousavi, A. Z. Genack, and A. B. Khanikaev, Nat. Mater. 15, 542 (2016).

${ }^{27}$ W.-J. Chen, M. Xiao, and C. T. Chan, Nat. Commun. 7, 13038 (2016).

${ }^{28}$ K. Lai, T. Ma, X. Bo, S. Anlage, and G. Shvets, Sci. Rep. 6, 28453 (2016).

${ }^{29}$ M. Hafezi, E. A. Demler, M. D. Lukin, and J. M. Taylor, Nat. Phys. 7, 907 (2011).

${ }^{30}$ S. Mittal, J. Fan, S. Faez, A. Migdall, J. M. Taylor, and M. Hafezi, Phys. Rev. Lett. 113, 087403 (2014).

${ }^{31}$ J. Noh, S. Huang, D. Leykam, Y. D. Chong, K. P. Chen, and M. C. Rechtsman, Nat. Phys. 13, 611-617 (2017).

${ }^{32}$ S. Weimann, M. Kremer, Y. Plotnik, Y. Lumer, S. Nolte, K. G. Makris, M. Segev, M. C. Rechtsman, and A. Szameit, Nat. Mater. 16, 433-438 (2017).

${ }^{33}$ W. Cai and V. Shalaev, Optical Metamaterials: Fundamentals and Applications (Springer, 2009).

${ }^{34}$ J. D. Joannopoulos, S. G. Johnson, J. N. Winn, and R. D. Meade, Photonic Crystals: Molding the Flow of Light (Princeton University Press, 2008).

${ }^{35}$ M. Decker, I. Staude, M. Falkner, J. Dominguez, D. N. Neshev, I. Brener, T. Pertsch, and Y. S. Kivshar, Adv. Opt. Mater. 3, 813-820 (2015).

${ }^{36}$ A. I. Kuznetsov, A. E. Miroshnichenko, M. L. Brongersma, Y. Kivshar, and B. Luk'yanchuk, Science 354, 846 (2016).

${ }^{37}$ I. Staude and J. Schilling, Nat. Photonics 11, 274 (2017).

${ }^{38}$ S. Kruk and Y. S. Kivshar, ACS Photonics 4, 2638 (2017).

${ }^{39}$ S. M. Kamali, E. Arbabi, A. Arbabi, and A. Faraon, Nanophotonics 7(6), 1041-1068 (2018).

${ }^{40}$ A. P. Slobozhanyuk, S. H. Mousavi, X. Ni, D. Smirnova, Y. S. Kivshar, and A. B. Khanikaev, Nat. Photonics 11, 130 (2017).

${ }^{41}$ T. Ma, A. B. Khanikaev, S. H. Mousavi, and G. Shvets, Phys. Rev. Lett. 114, $127401(2015)$.

${ }^{42}$ K. Y. Bliokh, F. J. Rodríguez-Fortuño, F. Nori, and A. V. Zayats, Nat. Photonics 9, 796 (2015).

${ }^{43}$ R. Alaee, M. Albooyeh, A. Rahimzadegan, M. S. Mirmoosa, Y. S. Kivshar, and C. Rockstuhl, Phys. Rev. B 92, 245130 (2015).

${ }^{44}$ S. H. Mousavi, A. B. Khanikaev, and Z. Wang, Nat. Commun. 6, 8682 (2015).

${ }^{45}$ D. A. Powell and Y. S. Kivshar, Appl. Phys. Lett. 97, 091106 (2010).

${ }^{46} \mathrm{M}$. Albooyeh, R. Alaee, C. Rockstuhl, and C. Simovski, Phys. Rev. B 91, 195304 (2015).

${ }^{47}$ V. Valuckas, R. Paniagua-Dominguez, Y. H. Fu, B. Luk'yanchuk, and A. I. Kuznetsov, Appl. Phys. Lett. 110, 091108 (2017). 